\begin{tabular}{|c|l|}
\hline Title & Mechanism of yellow luminescence in GaN at room temperature \\
\hline Author(s) & Matys, M.; A damowicz, B. \\
\hline Citation & $\begin{array}{l}\text { Journal of A pplied Physics, 121(6), 65104 } \\
\text { https://doi.org/10.1063/1.4975116 }\end{array}$ \\
\hline Issue Date & 2017-02-14 \\
\hline Doc URL & http://hdl.handle.net/2115/68307 \\
\hline Rights & $\begin{array}{l}\text { The following article appeared in Journal of A pplied Physics 121, 065104 (2017) and may be found at } \\
\text { http://aip.scitation.org/doi/10.1063/1.4975116. }\end{array}$ \\
\hline Type & article \\
\hline File Information & \begin{tabular}{l} 
1\% 2E4975116.pdf \\
\hline
\end{tabular} \\
\hline
\end{tabular}

Instructions for use 


\section{Mechanism of yellow luminescence in GaN at room temperature}

M. Matys and B. Adamowicz

Citation: Journal of Applied Physics 121, 065104 (2017); doi: 10.1063/1.4975116

View online: http://dx.doi.org/10.1063/1.4975116

View Table of Contents: http://aip.scitation.org/toc/jap/121/6

Published by the American Institute of Physics

\section{Articles you may be interested in}

Formation mechanism of gold-based and gold-free ohmic contacts to AIGaN/GaN heterostructure field effect transistors

Journal of Applied Physics 121, 065301065301 (2017); 10.1063/1.4975473

On the origin of interface states at oxide/III-nitride heterojunction interfaces

Journal of Applied Physics 120, 225305225305 (2016); 10.1063/1.4971409

Photo-Hall-effect study of excitation and recombination in Fe-doped GaN

Journal of Applied Physics 121, 065702065702 (2017); 10.1063/1.4975622

Au-free ohmic Ti/Al/TiN contacts to UID n-GaN fabricated by sputter deposition

Journal of Applied Physics 121, 065703065703 (2017); 10.1063/1.4975485

Three-dimensional cathodoluminescence characterization of a semipolar GalnN based LED sample

Journal of Applied Physics 121, 075702075702 (2017); 10.1063/1.4976204

Self-organized patterns of fullerene on molecular nanotemplate

Journal of Applied Physics 121, 054305054305 (2017); 10.1063/1.4975199

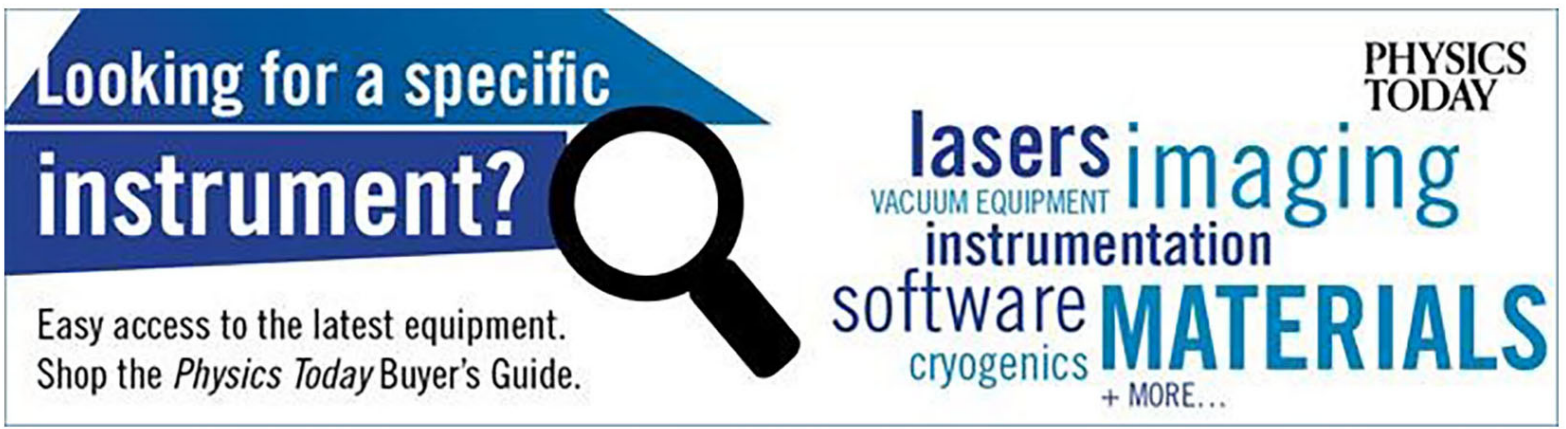




\title{
Mechanism of yellow luminescence in GaN at room temperature
}

\author{
M. Matys ${ }^{1,2, a)}$ and B. Adamowicz ${ }^{2}$ \\ ${ }^{1}$ Research Center for Integrated Quantum Electronics, Hokkaido University, Kita-13 Nishi-8, \\ Kita-ku 060-8628 Sapporo, Japan \\ ${ }^{2}$ Surface Physics and Nanostructures Department, Institute of Physics - CSE, Silesian University of Technology, \\ Konarskiego 22B, 44-100 Gliwice, Poland
}

(Received 16 October 2016; accepted 17 January 2017; published online 10 February 2017)

\begin{abstract}
We investigated the excitation intensity $(\Phi)$ dependent photoluminescence (PL), at room temperature (RT), from GaN-based metal-insulator-semiconductor structures under gate bias $\left(V_{G}\right)$ from accumulation to deep depletion resulting in variations of the space charge region width. We found that depending on $V_{G}$, different $\Phi$-dependencies of the YL band energy position (blueshift or redshift), shape (band enlargement or narrowing) and intensity (signal saturation) can be obtained. In order to explain such an unusual YL behavior, we developed a phenomenological PL model, which is based on the solution of the three-dimensional Poisson's equation, current continuity equations and rate equations, and which takes into account the grain structure of $\mathrm{GaN}$ layers and the contribution of interface regions into recombination processes. Our model reproduced well the experimental $\Phi$-dependencies of the YL band intensity. It also predicts that YL arises from the donor-acceptor pair (DAP) recombination in very limited areas (width of several nanometers) inside the depletion regions related to grain/grain interfaces and external crystal surfaces. On this basis, we showed that $V_{G^{-}}$-controlled $\Phi$ dependencies of the YL peak position and shape, can be well explained if we assume that YL is due to DAP-type transitions, in which the final state consists of the Coulomb interaction and strong interaction between the dipole moment of ionized DAP and the depletion region electric field. This recombination mechanism can play a significant role at RT, but should be negligible at low temperatures, where one can expect the significant reduction of interface barriers under illumination. Published by AIP Publishing. [http://dx.doi.org/10.1063/1.4975116]
\end{abstract}

\section{INTRODUCTION}

Photoluminescence (PL) was widely used as a key tool to study the material properties of wide band gap semiconductors (WBGS) like GaN, which plays a crucial role in technology of light-emitting ${ }^{1}$ and high-power electronic devices. $^{2}$ The most controversial and often observed in PL spectrum of $\mathrm{GaN}$ is the broad yellow luminescence (YL) band, whose source still remains a puzzle..$^{3-11}$ Generally, YL in $\mathrm{GaN}$ is interpreted in terms of two fundamental recombination mechanisms: (i) donor-acceptor pair (DAP) transitions, in which the final state (ionized pair) is determined by the Coulomb interaction ${ }^{12-15}$ and (ii) free-to-bound (eA) type transitions. ${ }^{3,16,17}$

However, in this work, we demonstrate that (1) YL in $\mathrm{GaN}$ at room temperature (RT) can arise as a result of DAPtype transitions, in which the final state is determined by not only the Coulomb interaction but predominately by the strong interaction between the dipole moment of the ionized DAP and the electric field in the depletion region, and (2) broadening of the YL band at RT can be due to the angular distribution of dipole moments with respect to the electric field. In this purpose, we carried out the systematic measurements of the excitation intensity $(\Phi)$ dependencies of PL spectra from GaN-based metal-insulator-semiconductor (MIS) structures at the different gate bias $\left(V_{G}\right)$ controlling the space charge region (SCR) width $\left(W_{S C R}\right)$. To the best of our knowledge, no

a)matys@rciqe.hokudai.ac.jp similar studies had been conducted for GaN. We observed that depending on $V_{G}$, different $\Phi$-dependencies of the YL band energy position (blueshift or redshift), shape (band enlargement or narrowing) and intensity (signal saturation) can be obtained. In order to explain such an unusual YL behavior, we developed a phenomenological model, which is based on the solution of the three-dimensional (3D) Poisson's equation, current continuity equations and rate equations, and which takes into account the grain structure of a GaN layer and the contribution of interface regions into the recombination processes.

\section{EXPERIMENT}

The schematic illustration of the investigated GaNbased MIS structure and gated-PL measurement is shown in Fig. 1. The MIS structures were fabricated using intentionally undoped n-GaN films with a free electron concentration $N_{d}=10^{17} \mathrm{~cm}^{-3}$ and thickness of $2.5 \mu \mathrm{m}$ grown by means of metalorganic chemical vapor deposition (MOCVD) technique on a sapphire substrate. The GaN layers were passivated with $\mathrm{Al}_{2} \mathrm{O}_{3}$ atomic layer deposition (ALD) insulating film (thickness of $20 \mathrm{~nm}$ ). In the passivation process, a twostep approach was applied, namely, at first, the coverage with a $10 \mathrm{~nm}$ thick $\mathrm{SiN}$ protection film deposited by electron cyclotron resonance chemical vapor deposition (ECR CVD) to avoid GaN damages during ohmic contact annealing, followed by removal of this film, and subsequent deposition of $\mathrm{Al}_{2} \mathrm{O}_{3}$. The ohmic contacts were ring-shaped $\mathrm{Ti} / \mathrm{Al} / \mathrm{Ti} / \mathrm{Au}$ 


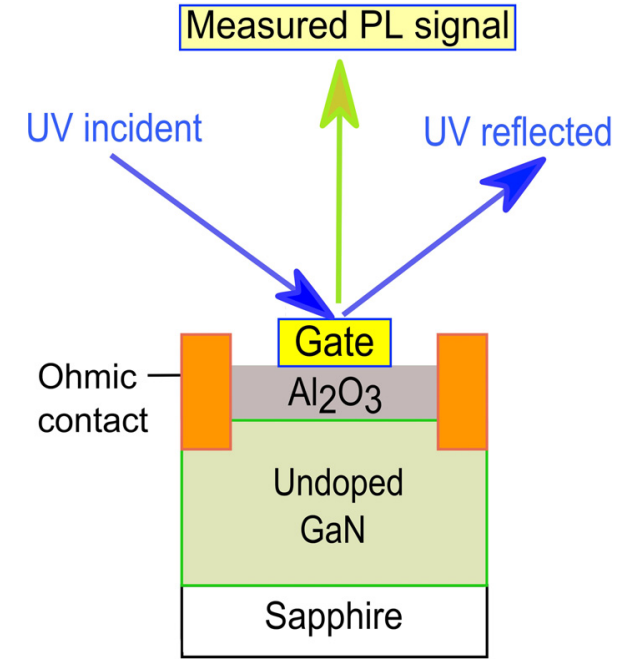

FIG. 1. Schematic illustration of the examined GaN- based MIS structure and gated-PL measurement. The focused light spot was limited to the gate area.

$(20 / 50 / 20 / 50 \mathrm{~nm})$ multilayers and the gate contacts were $\mathrm{Ni} /$ $\mathrm{Au}(50 \mathrm{~nm})$ circles with diameters from 200 to $500 \mu \mathrm{m}$. An ohmic annealing was carried out at $830^{\circ} \mathrm{C}$ for $2 \mathrm{~min}$ in $\mathrm{N}$ atmosphere. More details on fabrication and processing, one can find in Ref. 18. The steady-state PL measurements were carried out using a $325 \mathrm{~nm}$ continuous wave He-Cd laser at RT. The focused light spot was limited to the gate area and the $\Phi$ value (after absorption by the metal gate layer) was changed from $10^{14}$ to $10^{18}$ photon $/\left(\mathrm{cm}^{2} \mathrm{~s}\right)$. The electrical characteristics of the examined MIS structures were as follows: the flat band voltage $V_{F B}=0.2 \mathrm{~V}$ and the threshold voltage $V_{T}=-0.6 \mathrm{~V}$. The details of electrical measurements for these structures were reported in Ref. 19. In the gated PL experiment, we applied gate biases inducing $(i)$ accumulation $\left(V_{G} \geq V_{F B}\right)$, i.e., $V_{G}=1 \mathrm{~V}$, (ii) depletion $\left(V_{F B} \leq V_{G} \leq V_{T}\right)$, i.e., $V_{G}=-0.1,-0.3$, and $-0.5 \mathrm{~V}$, and (iii) deep depletion $\left(V_{G} \leq V_{T}\right)$, i.e., $V_{G}=-1,-2$, and $-3 \mathrm{~V}$.

\section{EXPERIMENTAL RESULTS}

\section{A. PL from unbiased MIS structure}

Before the gated-PL measurement, we performed the basic PL characterization of the GaN MIS structure with an unbiased gate, at RT. In Fig. 2, we presented the PL spectrum in the whole emission region under $\Phi=10^{18}$ photon/ $\left(\mathrm{cm}^{2} \mathrm{~s}\right)$. This spectrum consists of a broad yellow luminescence (YL) band in the range from 1.6 to $2.8 \mathrm{eV}$ and nearband edge (NBE) PL in the UV range from 3.2 to $3.6 \mathrm{eV}$. The integrated PL intensity $\left(I_{P L}\right)$ ratio of the NBE peak to the YL band is equal to about 0.2 . In addition, in the inset of Fig. 2, we displayed, for comparison, two normalized PL spectra in the UV range under low and high excitations, i.e., $\Phi=10^{15}$ photon $/\left(\mathrm{cm}^{2} \mathrm{~s}\right)$ and $\Phi=10^{18}$ photon $/\left(\mathrm{cm}^{2} \mathrm{~s}\right)$, respectively. One can note, that the peak position (PP) of NBE PL is the same for both excitations, which suggests that the possible heating of the sample due to UV- light irradiation is negligible.

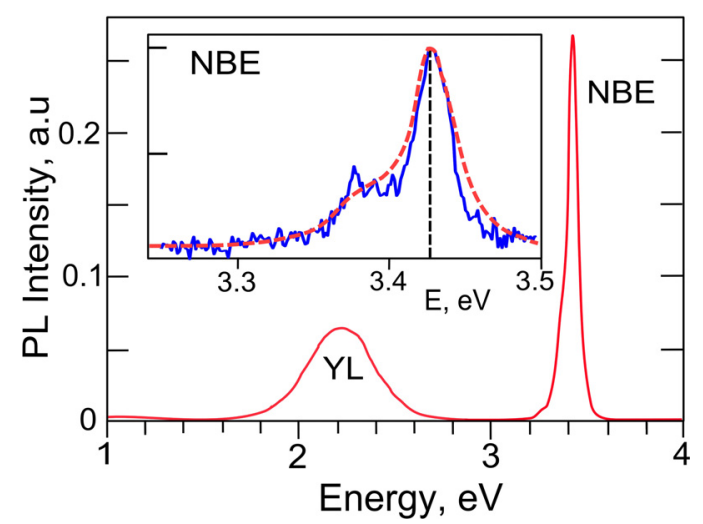

FIG. 2. Room-temperature photoluminescence spectrum of unbiased GaNbased MIS structure under $\Phi=10^{18}$ photon $/\left(\mathrm{cm}^{2} \mathrm{~s}\right)$. In the inset-normalized PL spectra in the UV range under $\Phi=10^{15}$ photon $/\left(\mathrm{cm}^{2} \mathrm{~s}\right)$ (solid line) and $\Phi=10^{18}$ photon $/\left(\mathrm{cm}^{2} \mathrm{~s}\right)$ (dashed line $)$.

\section{B. PL from biased MIS structure}

Fig. 3 presents the evolution of YL band with $\Phi$, recorded at RT, under biases from accumulation to deep depletion, i.e., $V_{G}=1,-0.5$, and $-3 \mathrm{~V}$. The $\Phi$-dependencies of PP and $I_{P L}$ of this band, at different $V_{G}$, are summarized in Fig. 4. Upon all applied biases, from $1 \mathrm{~V}$ to $-3 \mathrm{~V}$, when the structure was illuminated by $\Phi=10^{14}$ photon $/\left(\mathrm{cm}^{2} \mathrm{~s}\right)$ PP of YL was centered at the same energy of $2.2 \mathrm{eV}$ (Figs. 3 and 4(a)). Under the increasing $\Phi$, in the case of the structure biased to accumulation, we observed a shift of the YL band towards the lower energy (redshift) with narrowing of the full width at half maximum (FWHM) as shown in Figs. 3(a) and 4(a). On the other hand, in the case of the structures biased to depletion and deep depletion, the bias dependent and opposite shifts as well as the changes in FWHM of YL band with increasing $\Phi$ were obtained. Namely, under depletion conditions, the YL band is shifted to the higher energy (blueshift) with a widening of FWHM (shown in Figs. 3(b) and 4(a)) whereas under deep depletion conditions, the YL band exhibits a redshift and narrowing of FWHM (Figs. 3(b) and 4(a)). The blueshift is the largest for $V_{G}$ around $V_{T}$ and is gradually reduced for $V_{G}$ close to $V_{F B}$, whereas the redshift is the largest around $V_{G}=-3 \mathrm{~V}$ and decreases towards $V_{T}$. Furthermore, we observed the strong variations in the $\Phi$-dependencies of $I_{P L}$ of YL under different $V_{G}$. Namely, for more negative $V_{G}$, the saturation of YL band occurs under lower $\Phi$. In addition, the slope of $I_{P L}$ curves, in the weaker excitation range, is more pronounced at less negative $V_{G}$, as shown in Fig. 4(b). Moreover, we noticed that in some $\Phi$ ranges, the more negative $V_{G}$ inducing a widening of $W_{S C R}$ caused a dramatic increase of the intensity of YL band, which is totally in contradiction to the dead layer model, ${ }^{2}$ and some $\Phi$ ranges where the band intensity behaves upon changing $V_{G}$ according to the dead layer model.

It is evident that eA type transitions as well as DAP transitions, in which the final state is determined by the Coulomb interaction, cannot explain the behavior of $\mathrm{YL}$ band, in particular, the redshift of the band PP with increasing $\Phi$. On the other hand, the potential fluctuations ${ }^{20,21}$ due to the random distribution of charged defects could explain only some observations in our experiment, e.g., large shifts of PP. However, the presence of such fluctuations in undoped 
a)

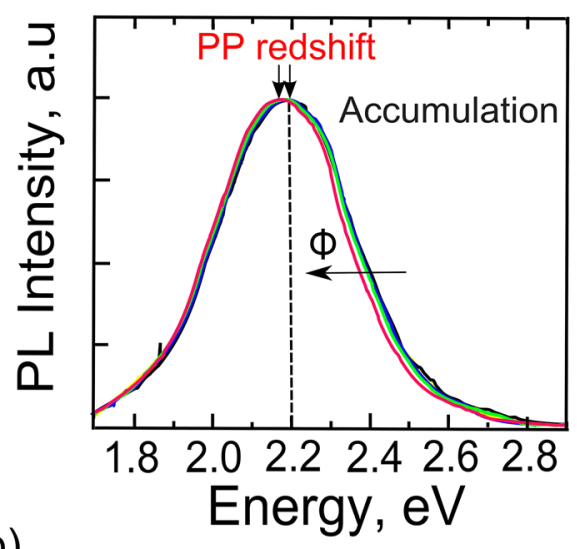

b)

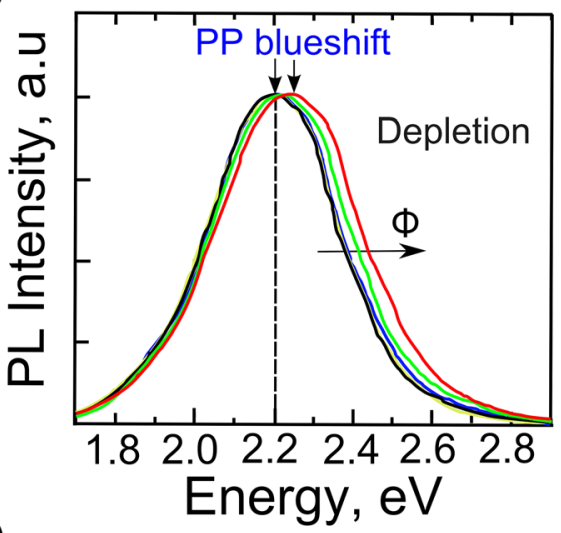

c)

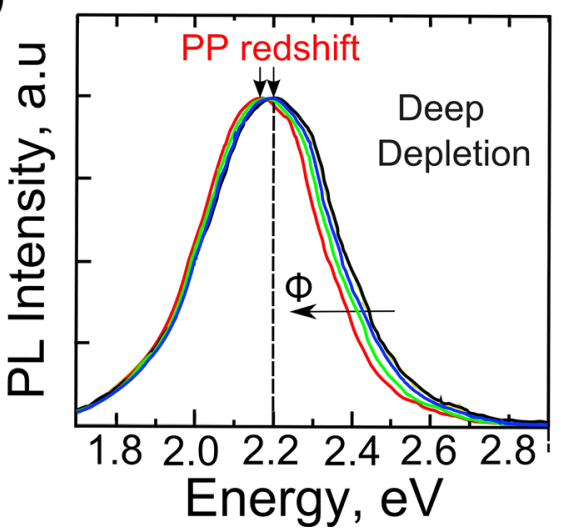

FIG. 3. Evolution of the normalized PL spectrum from a GaN based MIS structure with $\Phi$ increasing from $10^{14}$ to $10^{18}$ photon/ $\left(\mathrm{cm}^{2} \mathrm{~s}\right)$ with a step of one order of magnitude, at RT and under $V_{G}=1 \mathrm{~V}$ (accumulation) (a), $V_{G}=-0.5 \mathrm{~V}$ (depletion) (b), and $V_{G}=-3 \mathrm{~V}$ (c) (deep depletion).

n-GaN samples is of low probability. Furthermore, we excluded the surface related YL mechanisms ${ }^{22-25}$ because of an U-shaped continuum of non-radiative interface states existing at the $\mathrm{Al}_{2} \mathrm{O}_{3} / \mathrm{GaN}$ interface. ${ }^{19}$ Moreover, it is evident that the obtained dependencies of $I_{P L}$ cannot be explained by models based on the "dead layer" assumption (lack of radiative recombination in the depletion region). Therefore, an entirely new mechanism of YL is needed to explain our results.

\section{MODEL}

In order to clarify the mechanism of YL, we proposed the following model schematically illustrated in Fig. 5. a)
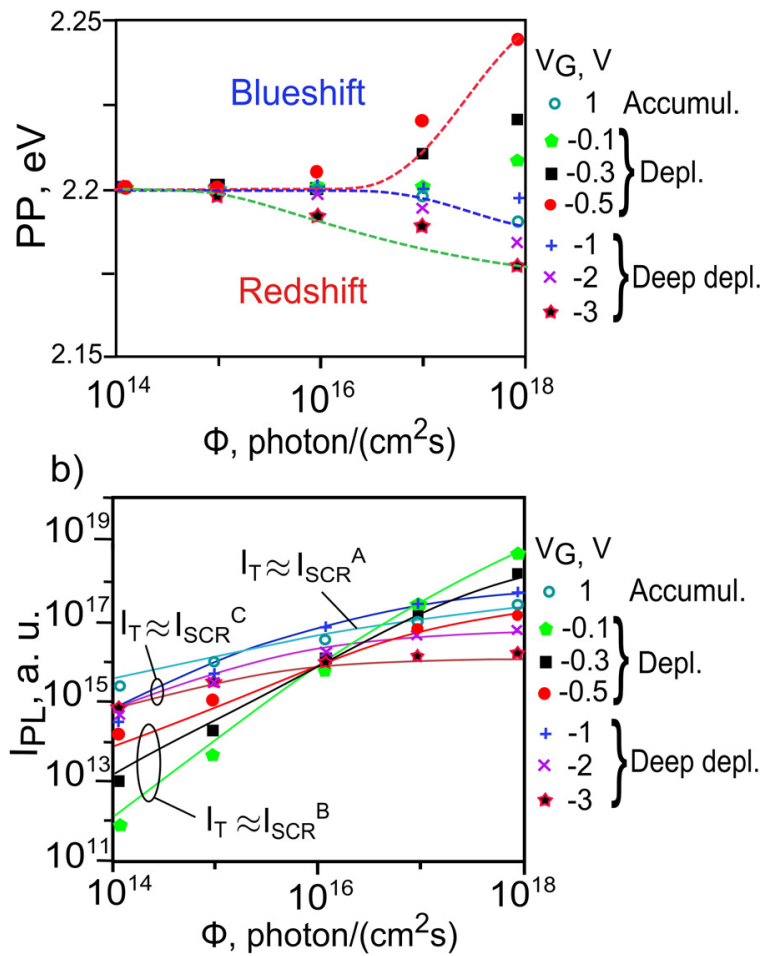

FIG. 4. PP (a) and $I_{P L}$ (b) of the YL band from GaN based MIS structure as a function of $\Phi$ at RT under different $V_{G}$. Dashed lines were calculated using Eq. (31). Solid lines are from a numerical solution of Eqs. (5)-(12) with the fitting parameters from Table I.

Let us consider a passivated semiconductor layer with the columnar grain structure, which is often observed in crosssectional transmission electron microscopy (TEM) micrographs $^{26}$ of GaN like in the image displayed in Fig. 5. The considered layer thickness is $\mathrm{L}$ and the length is equal to width $W=l^{0.5} \mathrm{~S}$, where $l$ is the number of grains, and $\mathrm{S}$ is the grain dimension. Then, let us assume that the layer contains: $(i)$ shallow donors (SD) with the concentration $N_{S D}$ and binding energy $E_{S D}$, (ii) deep acceptors (DA) with the concentration $N_{D A}$ and binding energy $E_{D A}$, (iii) non-radiative centers with the binding energy $E_{T}$ and (iv) interface states at the insulator/ semiconductor $(\mathrm{I} / \mathrm{S})$ interfaces with the density distribution $D_{I / S}(E)$ and at the grain-grain $(\mathrm{G} / \mathrm{G})$ interfaces with the density $D_{G / G}(E)$. Let us also assume that the interface states at $\mathrm{I} / \mathrm{S}$ and $\mathrm{G} / \mathrm{G}$ interfaces consist of both donor-like states distributed in the lower part of the band gap and acceptor-like ones in the upper part, which are separated by the charge neutrality level $E_{C N L}$ (for $\mathrm{GaN}, E_{C N L}=E_{C}-1.1 \mathrm{eV},{ }^{19}$ where $E_{C}$ is the bottom of the conduction band (CB)).

In the dark, the negatively charged acceptor-like interface states induce the band bending at $\mathrm{I} / \mathrm{S}$ and $\mathrm{G} / \mathrm{G}$ interfaces, as shown in the insets in Fig. 5. Upon the UV illumination, the excess carriers are generated at a rate $\mathrm{G}$ exponentially decayed from the $\mathrm{I} / \mathrm{S}$ interface according to the Lambert-Beer law, i.e., $G(x)=G(0) \exp \left(-\alpha_{a} \cdot x\right)$, where $\mathrm{G}(0)$ is the generation rate at the I/S interface, $\alpha_{a}=1.2 \times 10^{5}$ $\mathrm{cm}^{-1}$ is the absorption coefficient for $\mathrm{GaN}$ at $325 \mathrm{~nm}$, and $\mathrm{x}$ is the distance from the I/S interface. The excited carriers become separated in the depletion layer, i.e., electrons are 


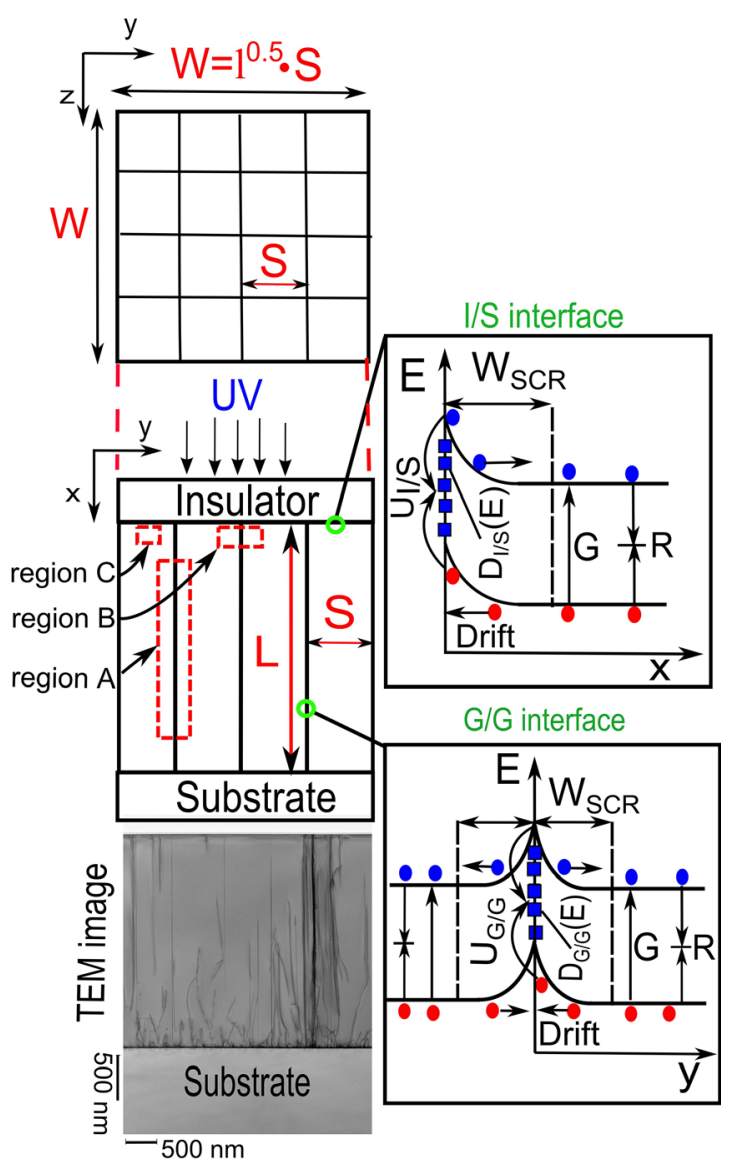

FIG. 5. Schematic illustration of an UV illuminated passivated semiconductor layer with the columnar grain structure, according to the cross-sectional TEM image of a GaN film in the studied MIS structure. In the insets - energy band diagrams of near $\mathrm{I} / \mathrm{S}$ and $\mathrm{G} / \mathrm{G}$ interface regions with $U_{I / S}, D_{I / S}(E)$ and $U_{G / G}, D_{G / G}(E)$, respectively, as well as with marked drift, generation $(\mathrm{G})$, and recombination $(\mathrm{R})$ processes.

repelled into the bulk whereas holes are attracted towards the interface (insets in Fig. 5). On the other hand, some holes are captured by donor-like interface states and cause the reduction of the negative interface charge. Both these processes result in the lowering of the interface band bending. Furthermore, the excess carriers recombine non-radiatively through the interface states at a rate $U_{I / S}$ and $U_{G / G}$ at I/S and $\mathrm{G} / \mathrm{G}$ interfaces, respectively, as presented in Fig. 5. According to the Shockley-Read-Hall (SRH) statistics, $^{27}$ $U_{I / S}$ is expressed by the following formula:

$$
U_{I / S}=\int_{E_{V}}^{E_{C}} \frac{\sigma_{n_{I / S}} \sigma_{p_{I / S}} v_{n} v_{p}\left(n_{I / S} p_{I / S}\right) D_{I / S}(E) d E}{\sigma_{n_{I / S}} v_{n}\left[n_{I / S}+n_{S}(E)\right]+\sigma_{p_{I / S}} v_{p}\left[p_{I / S}+p_{s}(E)\right]},
$$

where $E_{V}$ is the top of the valence band (VB); $\sigma_{n_{I / S}}$ and $\sigma_{p_{I / S}}$ are the cross sections for capturing electrons and holes, respectively, by interface states at the I/S interface; $v_{n}$ and $v_{p}$ are the thermal velocities of electrons and of holes, respectively; and $n_{I / S}$ and $p_{I / S}$ are the concentrations of electrons and holes, respectively, at the I/S interface; $n_{I / S}$ and $p_{I / S}$ are the concentrations of electrons and holes at the I/S interface, respectively, $n_{s}(E)=N_{C} \exp \left[\left(E-E_{C}\right) /(k T)\right]$ and $p_{s}(E)$ $=N_{V} \exp \left[\left(E_{V}-E\right) /(k T)\right]$. A similar relationship involving
TABLE I. The values of fitted parameters.

\begin{tabular}{lcc}
\hline \hline Parameter & Value & Unit \\
\hline$N_{D A}$ & $8 \times 10^{15}$ & $\mathrm{~cm}^{-3}$ \\
$C_{D A}$ & $10^{-7}$ & $\mathrm{~cm}^{3} / \mathrm{s}$ \\
$C_{S D}$ & $10^{-7}$ & $\mathrm{~cm}^{3} / \mathrm{s}$ \\
$B_{F B}$ & $10^{-10}$ & $\mathrm{~cm}^{3} / \mathrm{s}$ \\
$B_{D A P_{0}}$ & $10^{-10}$ & $\mathrm{~cm}^{3} / \mathrm{s}$ \\
$\tau$ & $10^{-7}$ & $\mathrm{~s}$ \\
$\mathrm{R}$ & $\approx 10$ & $\mathrm{~nm}$ \\
\hline \hline
\end{tabular}

the cross sections for electron $\left(\sigma_{n_{G / G}}\right)$ and hole capturing $\left(\sigma_{p_{G / G}}\right)$, respectively and $D_{G / G}(E)$, is valid for $U_{G / G}$.

The carriers can also recombine radiatively through free excitons and band-to-band transitions giving rise to the NBE PL via defects as well as non-radiatively through defects by a SRH mechanism ${ }^{27}$ with a rate $U_{S R H}$ given by the formula

$$
U_{S R H}=\frac{n p}{\tau_{n}\left[n+n_{1}\left(E_{T}\right)\right]+\tau_{p}\left[p+p_{1}\left(E_{T}\right)\right]},
$$

where $\tau_{n}$ and $\tau_{p}$ are the lifetimes of electrons and holes, respectively; $n$ and $p$ are the electron and hole concentrations, respectively; $n_{1}\left(E_{T}\right)=N_{C} \exp \left[\left(E_{T}-E_{C}\right) /(k T)\right]$ and $p_{1}\left(E_{T}\right)=N_{V} \exp \left[\left(E_{V}-E_{T}\right) /(k T)\right]$.

Moreover, let us assume that the radiative recombination via defects may occur by DAP-type transitions involving SD and DA and by eA type transitions involving $\mathrm{CB}$ and DA. Furthermore, according to Colbow, ${ }^{28}$ let us assume that the DAP recombination rate coefficient $B_{D A P}$ is dependent on the depletion region electric field strength $(F)$ and thus expressed by ${ }^{28}$

$$
B_{D A P}=\left(B_{D A P_{0}} / R_{1}\right)\left|1-8 \Theta R_{1}\right|^{-0.5} \exp (-\beta),
$$

where $B_{D A P_{0}}$ is the proportionality constant, $\Theta=q F a_{d} /$ $\left(2 E_{S D}\right), R_{1}=R / a_{d}$ where $R$ is the mean distance between DAPs and $a_{d}$ is the effective Bohr radius, $q>0$ is the elementary charge, and $\beta$ is expressed by

$$
\beta=\int_{\eta_{1}}^{2 R_{1}}\left(1-2 / \eta-4 / \eta^{2}-4 \Theta \eta\right)^{0.5} d \eta
$$

where $\eta_{1}$ is determined from a condition that the integrand in Eq. (4) is equal to zero. One can note that in the region where $F=0, B_{D A P}$ is approximately equal to $B_{D A P_{0}} \exp \left(-R_{1}\right)$, which is the probability of recombination in the ThomasHopfield model of DAP. ${ }^{29}$

The model equations are based on the 3D Poisson's equation, current continuity equations and rate equations describing the change of the concentration of defects at different charge states. The model equations in a semiconductor layer are as follows:

$$
\begin{aligned}
\nabla^{2} V & =-\frac{q}{\epsilon_{0} \epsilon_{S}}\left(p-n+N_{S D}^{+}-N_{D A}^{-}\right), \\
\frac{d n}{d t}= & \frac{1}{q} \nabla J_{n}+G+I_{S D} N_{S D}^{0}-B_{B} n p \\
& -U_{S R H}-C_{S D} n N_{S D}^{+}-B_{F B} n N_{D A}^{0},
\end{aligned}
$$




$$
\begin{gathered}
\frac{d p}{d t}=-\frac{1}{q} \nabla J_{p}+G+I_{D A} N_{D A}^{0}-B_{B} n p \\
-U_{S R H}-C_{D A} N_{D A}^{-} p, \\
J_{n}=-q n \mu_{n} \nabla V+q D_{n} \nabla n, \\
J_{p}=-q p \mu_{p} \nabla V-q D_{p} \nabla p, \\
\frac{d N_{D A}^{0}}{d t}=C_{D A} N_{D A}^{-} p-I_{D A} N_{D A}^{0}-B_{D A P} N_{D A}^{0} N_{S D}^{0} \\
-B_{F B} n N_{D A}^{0}, \\
\frac{d N_{S D}^{0}}{d t}=C_{S D} n N_{S D}^{+}-I_{S D} N_{S D}^{0}-B_{D A P} N_{D A}^{0} N_{S D}^{0},
\end{gathered}
$$

where $V$ is the electric potential, $\epsilon_{S}$ is the relative semiconductor permittivity, and $\epsilon_{0}$ is the vacuum permittivity; $N_{S D}^{0}$, $N_{S D}^{+}, N_{D A}^{0}$, and $N_{D A}^{-}$are the concentrations of defects at different charge states and $N_{S D}=N_{S D}^{+}+N_{S D}^{0}, N_{D A}=N_{D A}^{-}+N_{D A}^{0}$; $\mu_{n}$ and $\mu_{p}$ are the mobilities of electrons and holes, respectively, $k$ is the Boltzman constant, $D_{n}=\mu_{n} \frac{k T}{q}$ and $D_{p}=\mu_{p} \frac{k T}{q}$ are the diffusion constants for electrons and holes, respectively; $C_{D A}$ and $I_{D A}$ are the DA hole-capture and emission coefficients, respectively and $I_{D A}=C_{D A} N_{V} \exp \left(-\frac{E_{D A}}{k T}\right), C_{S D}$ and $I_{S D}$ are the SD electron-capture and emission coefficients, respectively and $I_{S D}=C_{S D} N_{C} \exp \left(-\frac{E_{S D}}{k T}\right), N_{C}$ and $N_{V}$ are the effective state densities in $\mathrm{CB}$ and $\mathrm{VB}$, respectively; $B_{F B}$ is the free-to-bound (FB) recombination coefficient for DA, $B_{B}$ is the coefficient for NBE recombination, which includes free exciton and band-to-band transitions.

The model equation in the insulator is the Laplace's equation:

$$
\nabla^{2} V=0
$$

The above Equations (5)-(12) are solved selfconsistently for the stationary case using the finite element method with Neumann type boundary conditions at the interfaces determined by the sheet charge density at the I/S interface $\left(Q_{I / S}\right)$ and $\mathrm{G} / \mathrm{G}$ interface $\left(Q_{G / G}\right)$ as well as by $U_{I / S}$ and $U_{G / G}$. For instance, at the I/S interface, the boundary conditions take the form

$$
\begin{gathered}
\epsilon_{0} \epsilon_{S} F_{\text {sem }}-\epsilon_{0} \epsilon_{I} F_{i n s}=Q_{I / S}, \\
J_{n}=-q U_{I / S}, \\
J_{p}=q U_{I / S},
\end{gathered}
$$

where $\epsilon_{I}$ is the insulator permittivity, subscripts "sem" and "ins" denote the semiconductor and the insulator, respectively. $Q_{I / S}$ is expressed by

$$
Q_{I / S}=\frac{q}{\epsilon_{0} \epsilon} \int_{E_{V}}^{E_{C N L}} D_{I / S}(E)\left(1-f_{I / S}\right) d E-\int_{E_{C N L}}^{E_{C}} D_{I / S}(E) f_{I / S} d E
$$

where $f_{I / S}$ is the occupation function of interface states at the I/S interface. $f_{I / S}$ is expressed by the following formula:

$$
f_{I / S}=\frac{\sigma_{n_{I / S}} n_{I / S}+\sigma_{p_{I / S}} p_{s}(E)}{\sigma_{n_{I / S}} v_{n}\left[n_{I / S}+n_{S}(E)\right]+\sigma_{p_{I / S}} v_{p}\left[p_{I / S}+p_{s}(E)\right]} .
$$

In the case of the MIS structure, we assumed that the gate is distributed over the entire insulator surface, and the equations were solved with the additional Dirichlet type boundary conditions, i.e., $V=V_{G}$ at the gate and $V=0$ at the ohmic contact. The solutions of the model equations are 3D spatial distributions of $V, n, p, N_{S D}^{0}, N_{S D}^{+}, N_{D A}^{0}$, and $N_{D A}^{-}$. On this basis, we calculated the distribution of the recombination rates of: $(i)$ DAP and FB processes related to DA as $U_{D A}=U_{F B}+U_{D A P}$, where $U_{F B}=B_{F B} n N_{D A}^{0}$ and $U_{D A P}=B_{D A P} N_{D A}^{0} N_{S D}^{0}$ and (ii) NBE recombination as $U_{N B E}=B_{B} n p$.

Then, we derived the number of photons per second emitted due to recombination through DA and due to NBE recombination from the whole sample $\left(I_{T}\right)$ and from different sample regions, as marked in the scheme in Fig. 5, namely, from SCR related to the $\mathrm{G} / \mathrm{G}$ interface (region $\mathrm{A})\left(I_{S C R}^{A}\right)$, $\mathrm{SCR}$ related to both the $\mathrm{G} / \mathrm{G}$ and $\mathrm{I} / \mathrm{S}$ interfaces (region $\mathrm{B}$ ) $\left(I_{S C R}^{B}\right)$, SCR related to the I/S interface (region C) $\left(I_{S C R}^{C}\right)$ and from the bulk region $\left(I_{B u l k}\right)$. For the calculation of $I_{T}$ we used the following relationship: $I_{T}=C_{R} \int_{0}^{L} \int_{0}^{W} \int_{0}^{W} U_{i} d x d y d z$, where $\mathrm{i}=\mathrm{DA}$ or NBE and $C_{R}$ is the geometrical factor related to the used measurement system. The values of $I_{S C R}^{A}, I_{S C R}^{B}, I_{S C R}^{C}$, and $I_{B u l k}$ were calculated similarly as $I_{T}$ with the integration limits determined by the corresponding SCR size. It is also evident that the following relationship is fulfilled: $I_{T}=I_{S C R}^{A}+I_{S C R}^{B}+I_{S C R}^{C}+I_{B u l k}$.

\section{COMPARISON WITH EXPERIMENT AND DISCUSSION}

First, we performed the theoretical calculations of $I_{T}$ vs. $\Phi$ under different $V_{G}$, at RT, using the developed phenomenological model described in Sec. IV and then compared, by means of fitting, the obtained $I_{T}(\Phi)$ dependencies with experimental $I_{P L}(\Phi)$ ones from Fig. 4(b). In the calculations, we used a set of model parameters related to both the bulk and surfaces/interfaces of the GaN MIS structure as well as the structure dimensions. The interface parameters were assumed according to the literature studies. Namely, the parameters of $\mathrm{Al}_{2} \mathrm{O}_{3} / \mathrm{GaN}$ interface, i.e., $D_{I / S}(E)=10^{11} \mathrm{~cm}^{-2} \mathrm{eV}^{-1}$ and $\sigma_{n_{I / S}}=\sigma_{p_{I / S}}=10^{-16} \mathrm{~cm}^{2}$, were recently obtained by our group using the photo-electric methods. ${ }^{19,30}$ The G/G interface parameters, i.e., $D_{G / G}(E) \approx 10^{12} \mathrm{~cm}^{-2} \mathrm{eV}^{-1}$ and $\sigma_{n_{G / G}}=\sigma_{p_{G / G}} \approx 10^{-15} \mathrm{~cm}^{2}$, were taken from the photoconductance studies. ${ }^{22,23,31}$ On the other hand, the grain dimension was $\mathrm{S}=500 \mathrm{~nm}$, as estimated from the cross-sectional TEM micrographs (Fig. 5), and the GaN layer thickness was $\mathrm{L}=2.5 \mu \mathrm{m}$. Furthermore, since the fitting procedure was not sensitive to the value $l>16$, we assumed $l=16$ in order to optimize the calculation time. Similarly, the values of $E_{D A}$ and $E_{T}$ had a negligible influence on the simulation results, because of the lack of emission of electrons or holes from these levels at RT, therefore $E_{D A}=E_{T}=1 \mathrm{eV}$ were arbitrarily introduced. We also assumed that $E_{S D}=30 \mathrm{meV}$ (which is a typical value for shallow donors in undoped $\mathrm{GaN}$ ) and $N_{S D}=N_{d}$. The value of the bimolecular recombination coefficient $B_{B}=10^{-8} \mathrm{~cm}^{3} / \mathrm{s}$ was taken from Ref. 32 . The material 
parameters of GaN were assumed according to Ref. 2, as follows: $\mu_{n}=1200 \mathrm{~cm}^{2} /(\mathrm{V} \mathrm{s}), \mu_{p}=10 \mathrm{~cm}^{2} /(\mathrm{V} \mathrm{s}), \epsilon_{S}=10.35$, $N_{C}=2.3 \times 10^{18} \mathrm{~cm}^{-3}$, and $N_{V}=1.8 \times 10^{19} \mathrm{~cm}^{-3}$. The other parameters, i.e., $N_{D A}, C_{D A}, B_{F B}, B_{D A P_{0}}, C_{S D}, R, \tau_{p}$, and $\tau_{n}$ were obtained from the fitting. Based on the recently reported first-principle calculations ${ }^{33,34}$ and electrical measurements, ${ }^{35,36}$ we were able to estimate fairly narrow ranges of reasonable values, in which the fitted parameters could be changed, i.e., $N_{D A}$ from $10^{14}$ to $10^{16} \mathrm{~cm}^{-3}, C_{D A}$ from $10^{-10}$ to $10^{-7} \mathrm{~cm}^{3} / \mathrm{s}$, and $C_{S D}$ from $10^{-10}$ to $10^{-7} \mathrm{~cm}^{3} / \mathrm{s}, B_{F B}$ from $10^{-13}$ to $10^{-10} \mathrm{~cm}^{3} / \mathrm{s}$ and $B_{D A P_{0}}$ from $10^{-13}$ to $10^{-10} \mathrm{~cm}^{3} / \mathrm{s}$. In the calculations we assumed, for simplicity, that $\tau_{p}=\tau_{n}=\tau$ is constant throughout the whole structure. The $\tau$ value was changed in a broad range from $10^{-4} \mathrm{~s}$ to $10^{-10} \mathrm{~s}$. It should be noted that the fitting can also be realized with $\tau_{p} \neq \tau_{n}$, however, the final results are close to those obtained in the case of $\tau_{p}=\tau_{n}$. The value of $\mathrm{R}$ should be larger than $a_{d} \approx 2.3 \mathrm{~nm}$, because Eq. (3) is valid for $R>a_{d}$. On the other hand, the average donor-acceptor distance $\mathrm{R}$ is less $\operatorname{than}^{20} N_{t}^{-1 / 3}$, where $N_{t}$ is the total impurity concentration (a sum of donor and acceptor concentrations). Since in our case (undoped ntype $\mathrm{GaN}$ ) the donor concentration is much higher than the acceptor concentration, thus $\mathrm{R}$ should be less than $N_{d}^{-1 / 3} \approx 20$ $\mathrm{nm}$. As a result, $\mathrm{R}$ was changed from $3 \mathrm{~nm}$ to $20 \mathrm{~nm}$.

The multi-parameter fitting of $I_{T}(\Phi)$ curves to the measured $I_{P L}(\Phi)$ dependencies was realized using a genetic algorithm $^{37}$ based method. The obtained values of fitted parameters are displayed in Table I. As it is evident from Fig. 4(b), the theoretical calculations of $I_{T}$ vs. $\Phi$ well reproduced the experimental $\Phi$-dependencies of $I_{P L}$ under various $V_{G}$. However, one can note that the values of fitted parameters $B_{F B}$ and $B_{D A P_{0}}$ are different (by about two orders of magnitude) than those reported in the literature, for example, by Reshchikov et al. ${ }^{38-40}$ On the other hand, the values of $C_{D A}$ and $C_{S D}$ of the order of $10^{-7} \mathrm{~cm}^{3} / \mathrm{s}$ are with a good agreement with the data by Reshchikov et al. ${ }^{38-40}$ It should also be noted that the determined $N_{D A}$ of the order of $10^{15} \mathrm{~cm}^{-3}$ is also consistent with the values reported by Reshchikov et al. ${ }^{38,39}$ Furthermore, the obtained $\tau=10^{-7} \mathrm{~s}$ lies in the range of the reported experimental values of $\tau$ for $\mathrm{GaN}^{41-43}$ from $10^{-9} \mathrm{~s}$ to $10^{-6} \mathrm{~s}$. The difference in values of $B_{F B}$ and $B_{D A P_{0}}$ parameters can result from the fact that in our model, we took into account the recombination in the depletion regions. It should also be noted that we do not claim that the values of parameters obtained from the fitting are unique or very precise. They should be rather treated as illustration that our phenomenological model with reasonable values can predict an unusual behavior of PL from GaN both under $V_{G}$ and $\Phi$ changing in wide ranges. In particular, the proposed model predicted the $V_{G}$-controlled saturation of YL intensity and a drastic increase of the YL band intensity vs. more negative $V_{G}$ (leading to enlargement of the depletion layer width), which is totally in contradiction with the dead layer model.

From the comparison of the calculated dependencies $I_{T}$ vs. $\Phi$ and experimental dependencies of $I_{P L}$ vs. $\Phi$ we found, as shown in Fig. 4(b), that $I_{T}$ is approximately equal to: $(i)$ $I_{S C R}^{A}$ (because of the inequality $\frac{I_{S C R}^{B}+I_{S C R}^{C}+I_{B u l k}}{I_{T}}<0.01$ ), when the structure is biased into accumulation, which would mean that YL comes from the depletion regions related to the $\mathrm{G} / \mathrm{G}$ interfaces, as it is seen from the cross-section distributions of $U_{D A}$ in Fig. 6(a), (ii) $I_{S C R}^{B}\left(\frac{I_{S C R}^{A}+I_{S C R}^{C}+I_{B u l k}}{I_{T}}<0.01\right)$, when the structure is biased to depletion, which would mean that YL originates from the depletion region parts common to $\mathrm{G} / \mathrm{G}$ and I/S interfaces, as shown in Fig. 6(b) and (iii) $I_{S C R}^{C}$ $\left(\frac{I_{S C R}^{A}+I_{S C R}^{B}+I_{B u l k}}{I_{T}}<0.01\right)$, when the structure is biased to deep depletion, which would mean that YL comes from the depletion regions related to the I/S interface, as can be seen from Fig. 6(c). Furthermore, the detailed analysis of $U_{D A}$ (Fig. 6) showed that in all these cases YL would not originate from
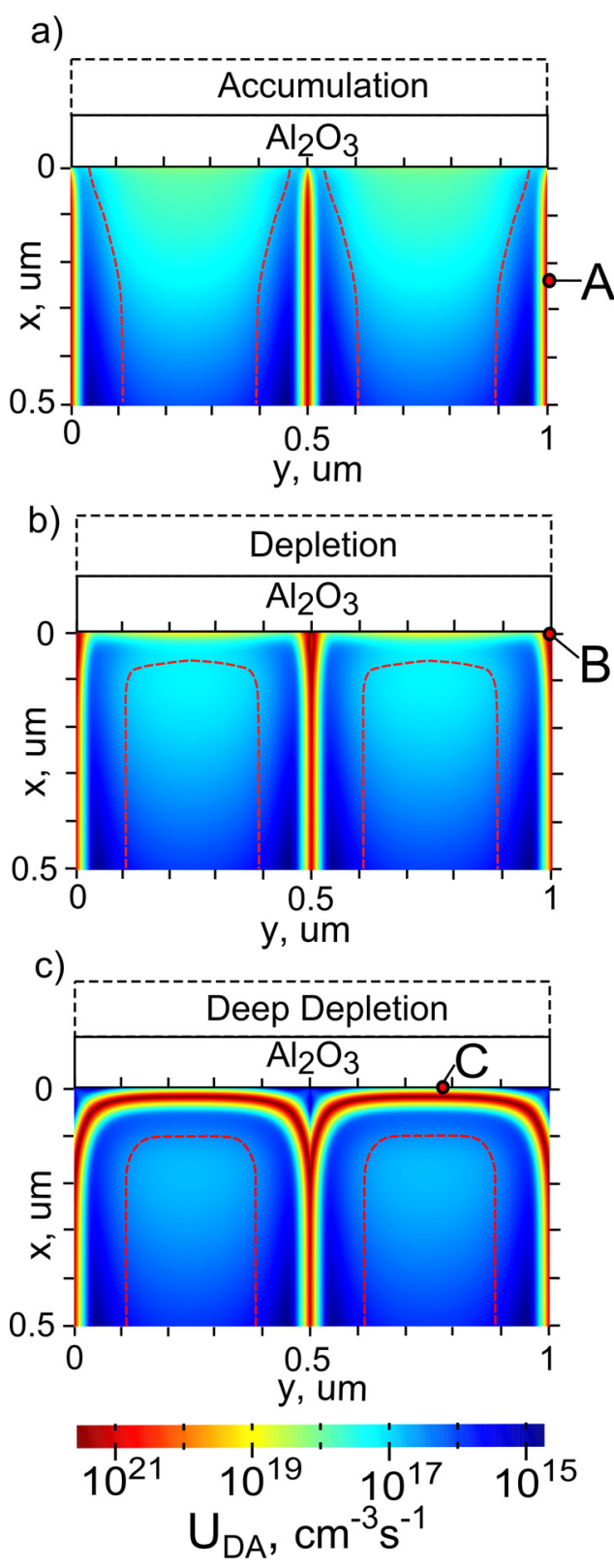

FIG. 6. Calculated cross-section distributions of $U_{D A}$ under $\Phi=10^{15}$ photon $/\left(\mathrm{cm}^{2} \mathrm{~s}\right)$ shown for two grains in the case of a structure biased to accumulation $\left(V_{G}=1 \mathrm{~V}\right)$ (a), depletion $\left(V_{G}=-0.5 \mathrm{~V}\right)$ (b) and deep depletion $\left(V_{G}=-1 \mathrm{~V}\right)(\mathrm{c})$. Dashed lines mark the border of depletion regions. The points $\mathrm{A}, \mathrm{B}$, and $\mathrm{C}$ indicate the locations where $F$ was calculated vs. $\Phi$, as shown in Fig. 12(b). 
the entire depletion region but mainly from the very limited narrow areas of these regions with a thickness of several nanometers. It should be noted that the calculations also indicate that in opposition to YL, NBE PL for all $V_{G}$ originates mainly from the areas outside the depletion regions, as it is seen from the cross-section distributions of $U_{N B E}$ in Fig. 7.

It is evident that the obtained result concerning YL is totally opposed to the conventional wisdom that PL mostly comes from areas outside the depletion regions. However, this result can be well understood based on simple rate equations (Eqs. (10) and (11)) describing the change of the concentration of defects at different charge states. Namely, in the stationary case, where $d N_{D A}^{0} / d t=0$ and $d N_{S D}^{0} / d t=0$,
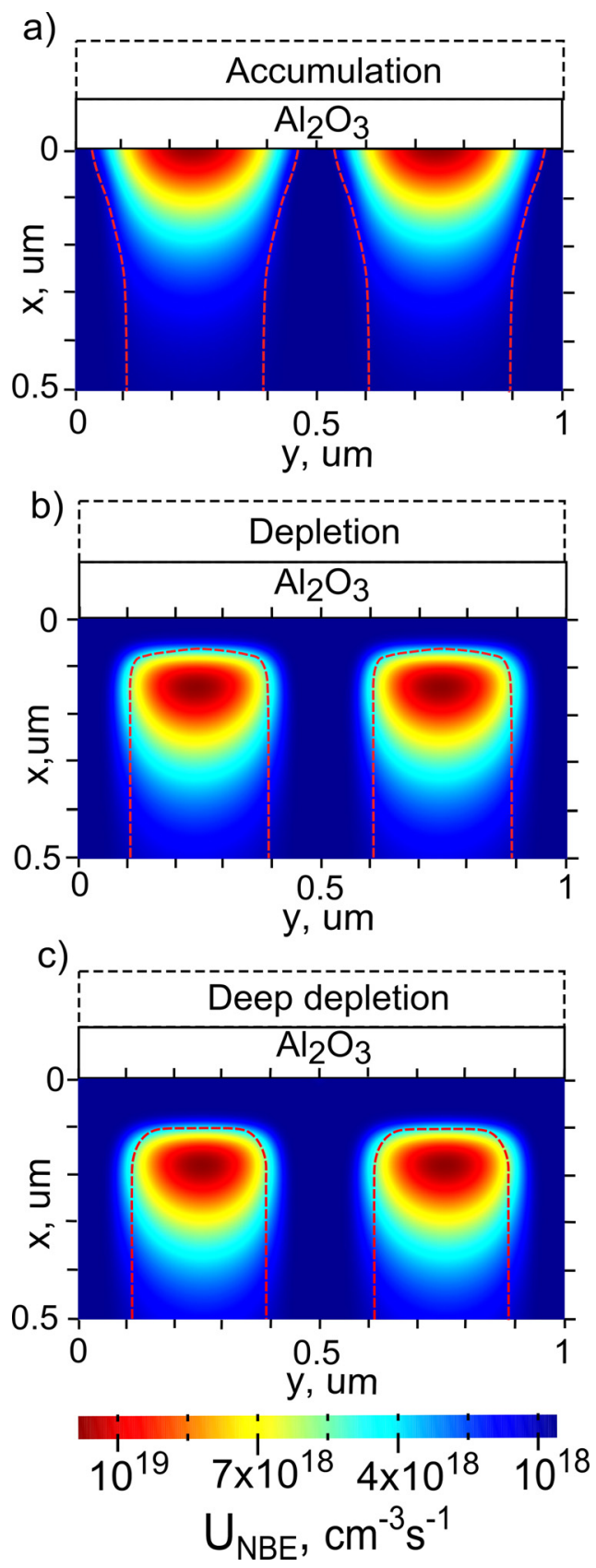

FIG. 7. Calculated cross-section distributions of $U_{N B E}$ under $\Phi=10^{15}$ photon/ $\left(\mathrm{cm}^{2} \mathrm{~s}\right)$ shown for two grains in the case of a structure biased to accumulation $\left(V_{G}=1 \mathrm{~V}\right)$ (a), depletion $\left(V_{G}=-0.5 \mathrm{~V}\right)$ (b) and deep depletion $\left(V_{G}=-1 \mathrm{~V}\right)(\mathrm{c})$. Dashed lines mark the border of depletion regions. and neglecting $I_{D A}$ in Eq. (10) (because of the lack of emission of holes from DA at RT), $N_{D A}^{0}$ and $N_{S D}^{0}$, from Eqs. (10) and (11), can be expressed as follows:

$$
\begin{aligned}
N_{S D}^{0} & =\frac{C_{S D} N_{S D} n}{I_{S D}+B_{D A P} N_{D A}^{0}+C_{S D} n}, \\
N_{D A}^{0} & =\frac{C_{D A} N_{D A} p}{C_{D A} p+B_{F B} n+B_{D A P} N_{S D}^{0}} .
\end{aligned}
$$

It can be easily shown that in the denominator in Eq. (18), the following relationship is fulfilled at RT: $I_{S D} \gg B_{D A P} N_{D A}^{0}+C_{S D} n$. Namely, at this condition, for $E_{S D}=30 \mathrm{meV}, \exp \left(-\frac{E_{S D}}{k T}\right) \approx 1 / 3$ and thus $I_{S D} \approx C_{S D} N_{C} / 3$. Therefore, we have that $N_{C} \gg 3\left(B_{D A P} N_{D A}^{0} / C_{S D}+n\right)$. Because $C_{S D}>B_{D A P}$ and $N_{D A}^{0} \ll N_{d}$ for n-type GaN, we obtained that $B_{D A P} N_{D A}^{0} / C_{S D} \ll N_{d}$. Furthermore, it is evident that $n \leq N_{d}$. Taking into account that in our case $3 N_{d} \ll 0.5$ $N_{C}$, we can write that $3\left(B_{D A P} N_{D A}^{0} / C_{S D}+n\right) \ll N_{C}$, which ends the proof. On this basis, Eq. (18) takes the form

$$
N_{S D}^{0} \approx \frac{C_{S D} N_{S D} n}{I_{S D}} .
$$

In $\mathrm{GaN}$, there exists high interface electric fields of the order of magnitude of $10^{5} \mathrm{~V} / \mathrm{cm}$. Such fields strongly attract almost all photo-holes towards the interfaces and repel electrons from the interfaces towards the bulk. In consequence, a very high $p$ and $n$ gradients between the interface and the bulk are induced, i.e., negligible/high $p$ in the bulk/near the interface, respectively and high/negligible $n$ in the bulk/near the interface, respectively, as shown in Fig. 8(a). Due to such distributions of $p$ and $n$, we can distinguish two regions (Fig. $8(\mathrm{a})$ ), namely, region I, extending from the interface to the point $x_{1}$, in which $n \ll p$ and region II, extending from $x_{2}$ to the bulk, in which $n \gg p$. It should be noted that both $x_{1}$ and $x_{2}$ points are located inside the depletion layer.

For region I, we can show that the following inequality (see the denominator in Eq. (19)) is fully satisfied:

$$
C_{D A} p \gg B_{F B} n+B_{D A P} N_{S D}^{0}
$$

Namely, the above inequality can be written as follows:

$$
p \gg B_{F B} n / C_{D A}+B_{D A P} N_{S D}^{0} / C_{D A} \text {. }
$$

Because $C_{D A}>B_{F B}$, then $B_{F B} n / C_{D A}<n$. On the other hand, using $I_{S D} \approx C_{S D} N_{C} / 3$ and Eq. (20), we can write that $B_{D A P} N_{S D}^{0} / C_{D A} \approx 3 B_{D A P} N_{S D} n /\left(C_{D A} N_{C}\right)$. From the fact that $C_{D A}>B_{D A P}$ and $3 N_{S D}<N_{C}$, it follows that $3 B_{D A P} N_{S D} n /$ $\left(C_{D A} N_{C}\right)<n$. Therefore, we find that $B_{F B} n / C_{D A}+B_{D A P}$ $N_{S D}^{0} / C_{D A}<2 n$. Because $2 n \ll p$ ( $p$ is several orders larger than $n$ in region I) thus $p \gg B_{F B} n / C_{D A}+B_{D A P} N_{S D}^{0} / C_{D A}$. On this basis, Eq. (19) in region I takes the simple form

$$
N_{D A}^{0} \approx N_{D A} .
$$

This means that in region I, all DA are neutral as can be seen in Fig. 8(a), which shows the calculated depth distribution of $N_{D A}^{0}$. 
a)

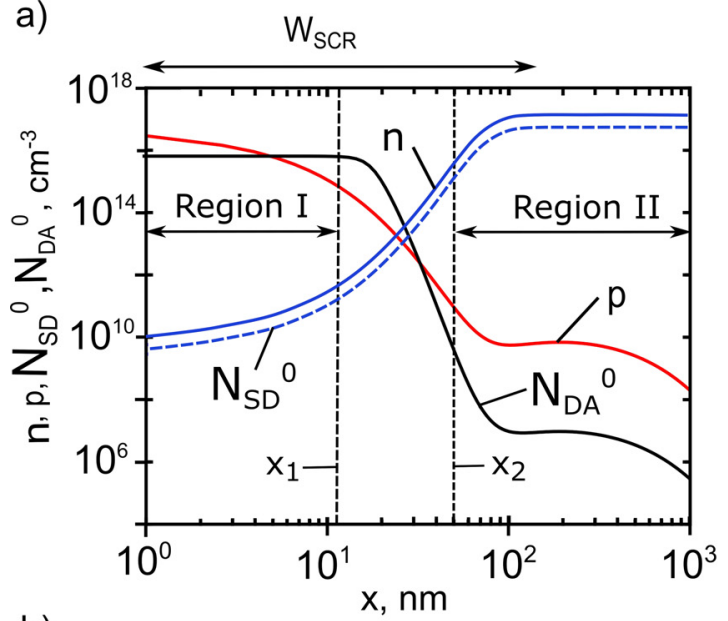

b)

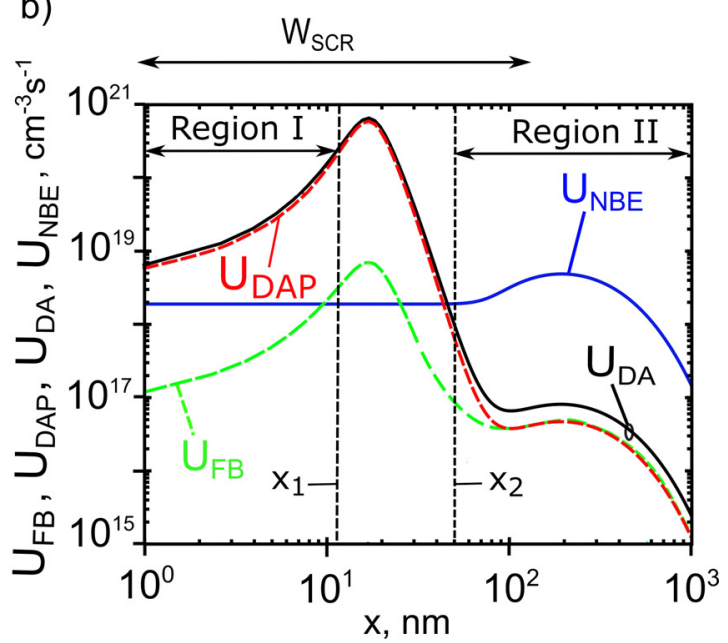

FIG. 8. Calculated in-depth distributions, from the I/S interface towards the bulk, of: $N_{D A}^{0}$, p, n, $N_{S D}^{0}$ (a), $U_{D A}, U_{F B}, U_{D A P}$, and $U_{N B E}$ (b). $V_{G}=-1 \mathrm{~V}$ and $\Phi=10^{15}$ photon $/\left(\mathrm{cm}^{2} \mathrm{~s}\right)$.

For region II $(n \gg p)$, we can prove that opposite relationship $p \ll B_{F B} n / C_{D A}+B_{D A P} N_{S D}^{0} / C_{D A}$ is satisfied. Namely, assuming that $B_{F B} \geq 10^{-12} \mathrm{~cm}^{3} / \mathrm{s}, \quad B_{D A P}$ $\geq 10^{-12} \mathrm{~cm}^{3} / \mathrm{s}$, and $C_{D A} \leq 10^{-7} \mathrm{~cm}^{\frac{3}{3}} / \mathrm{s}$, we obtain that $B_{F B} n / C_{D A} \geq 10^{-5} n$ and $B_{D A P} N_{S D}^{0} / C_{D A} \geq 10^{-5} N_{S D}^{0}$. Taking into account that in region II $10^{-5} n \gg p$ and $10^{-5} N_{S D}^{0} \gg p$, we find that $B_{F B} n / C_{D A}+B_{D A P} N_{S D}^{0} / C_{D A} \gg 2 p$, which ends the proof. Using this inequality and Eq. (20), Eq. (19) in region II can be approximately written as follows:

$$
N_{D A}^{0} \approx \frac{C_{D A} N_{D A} N_{C}}{B_{F B} N_{C}+3 B_{D A P} N_{S D}} \frac{p}{n} .
$$

From the above equation, it follows that $N_{D A}^{0}$ in region II strongly decreases, as shown in Fig. 8(a), first due to the rapid decrease of $p$ and increase of $n$ and subsequently due to the constant $n$ and decrease of $p$.

Taking into account Eqs. (20), (23), and (24), we can express $U_{D A P}$ and $U_{F B}$ as follows.

In region I:

$$
\begin{gathered}
U_{F B}=B_{F B} n N_{D A}^{0} \approx B_{F B} N_{D A} n \\
U_{D A P}=B_{D A P} N_{D A}^{0} N_{S D}^{0} \approx \frac{3 B_{D A P} N_{D A} N_{S D}}{N_{C}} n .
\end{gathered}
$$

In region II:

$$
\begin{gathered}
U_{F B} \approx \frac{B_{F B} C_{D A} N_{D A} N_{C}}{B_{F B} N_{C}+3 B_{D A P} N_{S D}} p, \\
U_{D A P} \approx \frac{3 B_{D A P} N_{S D} C_{D A} N_{D A}}{N_{C} B_{F B}+3 B_{D A P} N_{S D}} p .
\end{gathered}
$$

The above Eqs. (25)-(28) indicate that $U_{F B}, U_{D A P}$, and thus also $U_{D A}=U_{D A P}+U_{F B}$ increases, like $n$, from the interface to the point $x_{1}$, and strongly decreases, like $p$, from the point $x_{2}$ to the bulk, as shown in Fig. 8(b). From this it follows that $U_{F B}, U_{D A P}$, and $U_{D A}$ should reach maximum in the depletion layer in the range $\left\langle x_{1}, x_{2}\right\rangle$, as in Fig. 8(b). In consequence, almost all emissions related to DA will essentially come from the near interface region. Furthermore, it should be noted that the correlated increasing $n$ and decreasing $p$ in the range from the interface up to the point $x_{1}$ causes that $U_{N B E}$ remains almost constant in this region, as shown in Fig. 8(b). On the other hand, the stronger increasing $\mathrm{n}$ than decreasing $p$ in the region located deeper gives that $U_{N B E}$ rises up to the point beneath the depletion region, where it reaches the maximum (Fig. 8(b)) As a result, the NBE emission mostly comes from outside of the depletion regions.

From the performed analysis it follows that $U_{F B}, U_{D A P}$, and $U_{D A}$ always reach a maximum inside the depletion region independent of the $B_{F B}$ value (in the range $10^{-10} \mathrm{~cm}^{3} / \mathrm{s}$ $\left.\geq B_{F B} \geq 10^{-12} \mathrm{~cm}^{3} / \mathrm{s}\right), B_{D A P}\left(10^{-10} \mathrm{~cm}^{3} / \mathrm{s} \geq B_{D A P} \geq 10^{-12} \mathrm{~cm}^{3} / \mathrm{s}\right)$, $C_{D A}\left(10^{-7} \mathrm{~cm}^{3} / \mathrm{s} \geq C_{D A} \geq 10^{-9} \mathrm{~cm}^{3} / \mathrm{s}\right)$, and $C_{S D}\left(10^{-7} \mathrm{~cm}^{3} / \mathrm{s}\right.$ $\geq C_{S D} \geq 10^{-9} \mathrm{~cm}^{3} / \mathrm{s}$ ) if the largely non-uniform distributions of $n$ and $p$ exist, as in Fig. 8(a). In other words, $U_{F B}, U_{D A P}$, and $U_{D A}$ exhibit the maximum value in the depletion layer solely due to non-uniform $p$ and $n$ distributions induced by high electric fields. The $B_{F B}, B_{D A P}, C_{D A}$, and $C_{S D}$ parameters can only influence the maximum intensity of the mentioned rates. This means that exclusively high electric fields present in $\mathrm{GaN}$ at $\mathrm{RT}$ would be responsible for the localization of YL maximum in depletion regions. In addition, since $B_{D A P}$ is enhanced by $F$ [see Eqs. (3) and (4)], thus $U_{D A P} \gg U_{F B}$ in the depletion regions, as illustrated in Fig. 8(b). This means that the YL band would mainly be caused by DAP type transitions.

Based on the above results, we can explain the obtained relationship between $I_{T}$ and other components $I_{S C R}^{A}, I_{S C R}^{B}$, and $I_{S C R}^{C}$. Namely, in the case of the structures biased into accumulation, the photo-holes are collected near the $\mathrm{G} / \mathrm{G}$ interface, as shown in Fig. 9(a). As a result, the $U_{D A}$ maximum is also located in the depletion region related to the $\mathrm{G} / \mathrm{G}$ interface (Fig. 6(a)) and thus $I_{T} \approx I_{S C R}^{A}$ (Fig. 4(b)). On the other hand, when the structures are biased to depletion, the photo-holes are gathered mainly close to the $\mathrm{G} / \mathrm{G}$ and $\mathrm{I} / \mathrm{S}$ interfaces (Fig. 9(b)). In consequence, the $U_{D A}$ maximum is located in the part of the depletion region common to both these interfaces (Fig. 6(b)) and thus $I_{T} \approx I_{S C R}^{B}$ (Fig. 4(b)). Furthermore, under deep depletion, the photo-holes are mostly collected near the I/S interface (Fig. 9(c)) and thus the $U_{D A}$ maximum is placed in the depletion region (Fig. 6(c)) related to this interface resulting in $I_{T} \approx I_{S C R}^{C}$ (Fig. 4(b)).

From the above results it follows that $U_{D A}$ is totally governed by the carrier distribution in the depletion region. 
a)
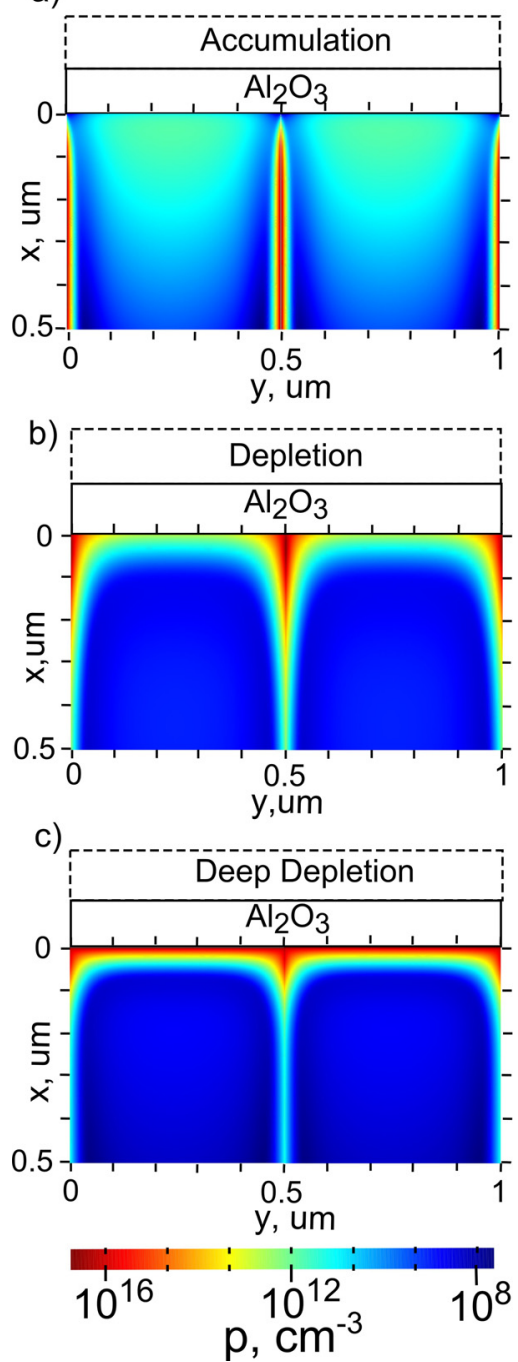

FIG. 9. Calculated cross-section distributions of $\mathrm{p}$ under $\Phi=10^{15}$ photon/ $\left(\mathrm{cm}^{2} \mathrm{~s}\right)$ shown for two grains in the case of a structure biased into accumulation $\left(V_{G}=1 \mathrm{~V}\right)$ (a), depletion $\left(V_{G}=-0.5 \mathrm{~V}\right)$ (b) and deep depletion $\left(V_{G}=-1 \mathrm{~V}\right)(\mathrm{c})$.

It would be a reason that for various $V_{G}$, we obtained significantly different $\Phi$-dependencies of $I_{P L}$ for YL. For example, $V_{G}=-1 \mathrm{~V}$ induces $U_{D A}$ with the maximum located close to the interface (case 1) whereas $V_{G}=-3 \mathrm{~V}$ induces $U_{D A}$ with the maximum located farther from the interface (case 2) as shown in Fig. 10. This is because of the weaker attraction of holes and weaker repelling electrons from the interface in case 1 than in case 2 . In case 1 , the maximum value of $U_{D A}$ saturates at higher $\Phi$ than in case 2, as shown in Fig. 10. As a consequence, $I_{P L}$ of YL saturates at higher $\Phi$ in case 1 than in case 2 (as illustrated in Fig. 4(b)). The different behavior of $U_{D A}$ maximum vs. $\Phi$ can be well understood in terms of the position of the hole quasi-Fermi level at the interface $\left(E_{f p s}\right)$. Namely, in case $1, E_{f p s}$ is at a larger distance from $E_{V}$ than in case 2, as shown in Fig. 11(a) and therefore a relatively higher $\Phi$ is necessary for $E_{f p s}$ to reach $E_{V}$. While $E_{f p s}$ approaches $E_{V}, p$ in the depletion region (Fig. 11(b)) and consequently the $U_{D A}$ maximum saturates at a higher $\Phi$ in case 1 compared to case 2 .

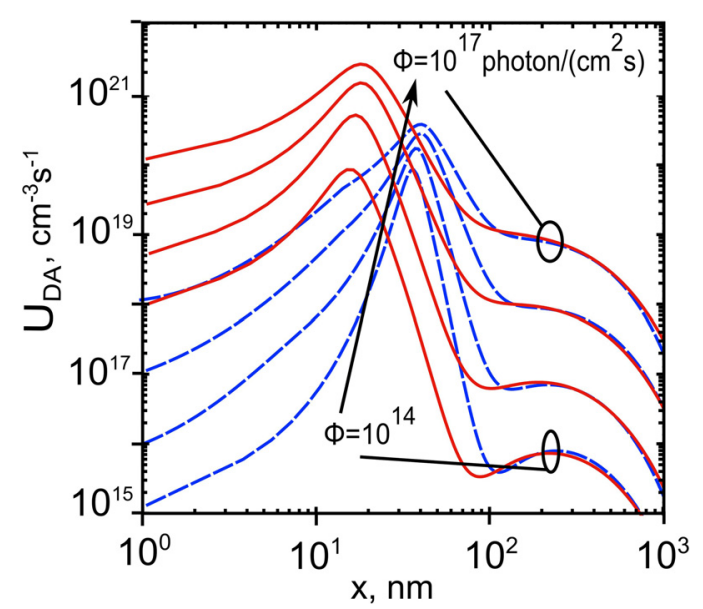

FIG. 10. Calculated in-depth distributions, from I/S interface towards the bulk, of $U_{D A}$ as a function of $\Phi$ with a step of one order of magnitude. Solid lines correspond to $V_{G}=-1 \mathrm{~V}$ and dashed lines to $V_{G}=-3 \mathrm{~V}$.

Our model predicted that YL arises from the DAP recombination in very limited areas inside the depletion regions. On this basis, we concluded that both PP and FWHM of YL can be dependent on $F$. This is because a photon emitted from DAP recombination under the field $F$ has the energy dependent on this field. It results from the interaction between $F$ and the dipole moment of the ionized DAP created after recombination involving a neutral DAP. ${ }^{28}$ The

a)

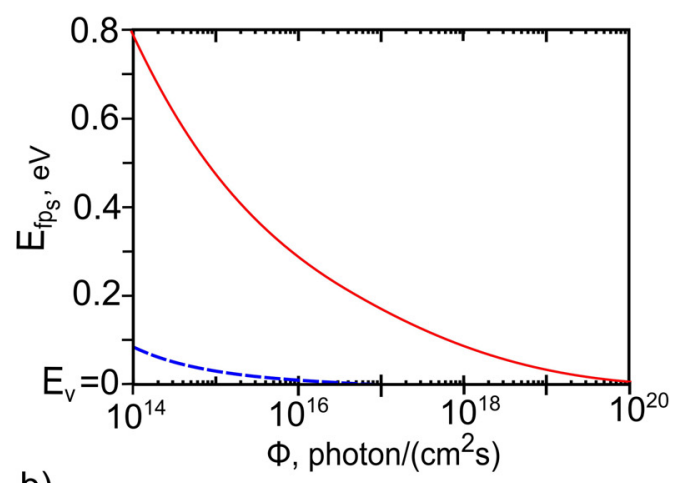

b)

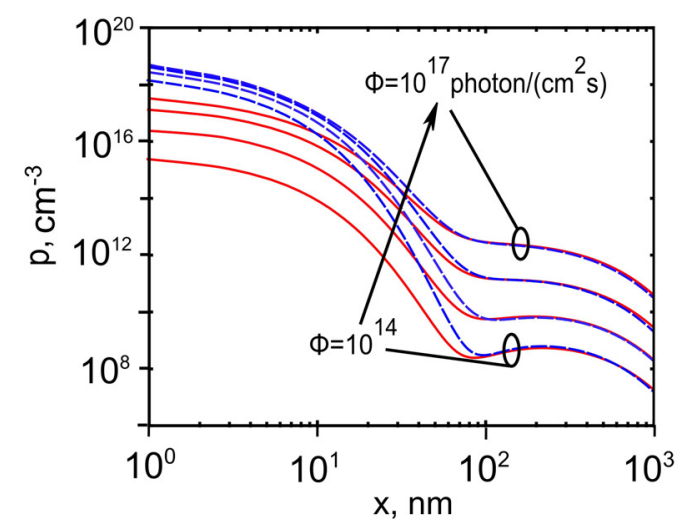

FIG. 11. Calculated dependencies of $E_{f p s}$ vs. $\Phi$ (a); in-depth distributions from I/S interface of $\mathrm{p}$ as a function of $\Phi$ with a step of one order of magnitude (b). Solid lines are for $V_{G}=-1 \mathrm{~V}$ and dashed lines for $V_{G}=-3 \mathrm{~V}$. 
emission energy $\left(E_{e m}\right)$ of DAP recombination in the depletion region can be expressed by the relationship ${ }^{28}$

$$
E_{\text {em }}=E_{g}-E_{D A}-E_{S D}+E_{C o u l}+q F R \cos \alpha,
$$

where $E_{g}$ is the energy band gap, $E_{\text {Coul }}$ is the Coulomb interaction, and $\alpha$ is the angle between the DAP dipole moment and $F$, and $\alpha$ takes values in the range ${ }^{28}-\pi / 2<\alpha \leq \pi / 2$. Thus, due to the dependence of $E_{e m}$ on $\alpha$, the YL bandwidth should be broadened by an amount $\Delta E$ approximately equal to ${ }^{28}$

$$
\Delta E \approx 0.5 q F R
$$

Therefore, according to Eqs. (29) and (30), one can expect that PP and FWHM of YL should exhibit a linear blueshift and increasing FWHM with the rising $F$ and, on the contrary, a linear redshift and decreasing FWHM with the reduced $F$.

In order to prove the linear relationships between YL parameters and $F$, we converted the measured PP dependencies vs. $\Phi$ (Fig. 4(a)) into the dependencies vs. $F$ (Fig. 12(a)). In the converting procedure we used the $F$ values calculated vs. $\Phi$, at the points A, B, and C, as shown in Fig. 6. The calculated dependencies $F$ vs. $\Phi$ are shown in Fig. 12(b). From this analysis, we obtained the striking correlation between PP and $F$, i.e., we found that all the data points from the drastically differing dependencies of PP after converting lie at a common straight line with a positive slope. These results excellently confirm the predicted dependencies of PP against $F$ (see Eq. (29)) and also the quantitative analysis of $I_{P L}$ (Fig. 4(b)) by the proposed model. Consequently, we can express $P P$ of YL as follows:

\section{a)}

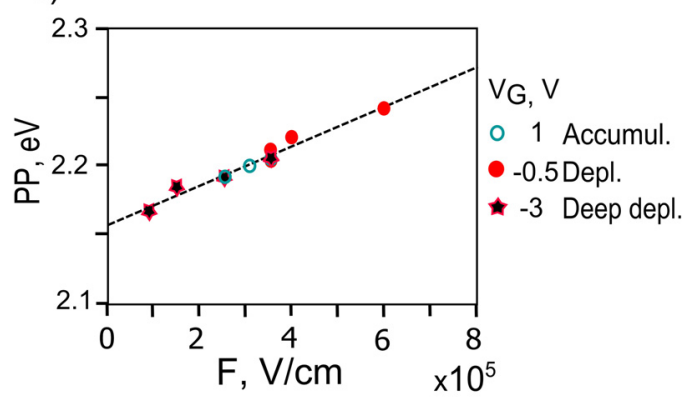

b)

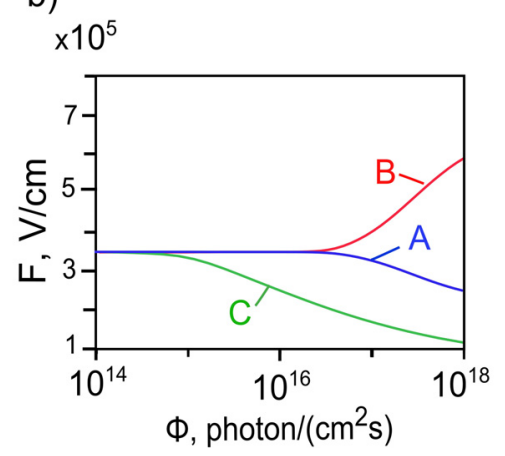

FIG. 12. Dependencies of PP vs. F (a) obtained after converting measured PP ( $\Phi)$ dependencies from Fig. 4(a). The $F$ values were obtained from the calculated dependencies $F(\Phi)$ for different $V_{G}(\mathrm{~b})$. The curves $\mathrm{A}\left(V_{G}=1 \mathrm{~V}\right)$, $\mathrm{B}(-0.5 \mathrm{~V})$, and $\mathrm{C}(-3 \mathrm{~V})$ were calculated at points $\mathrm{A}, \mathrm{B}$, and $\mathrm{C}$, respectively, from Fig. 6. Dashed line is the best fit curve: $P P=a F+b$, where $a=15 \times 10^{-10} \mathrm{~cm} / \mathrm{q}$ and $b=2.16 \mathrm{eV}$.

$$
P P=a F+b,
$$

where $a=15 \times 10^{-10} \mathrm{q} \mathrm{cm}$ and $b=2.16 \mathrm{eV}$.

It should be noted that when $F=0$ (lack of internal electric fields), PP of the YL band should be located at $2.16 \mathrm{eV}$. By analogy to Eq. (29), the constant $a$ in Eq. (31) should be directly proportional to $\mathrm{R}$. Thus, we can estimate $\mathrm{R}$ as follows: $\mathrm{R} \gtrsim a / q=9 \mathrm{~nm}$. It should be noted that this value is in a good agreement with the value of $\mathrm{R}$ (Table I) independently determined from the fitting of the $I_{P L}(\Phi)$ dependencies. This finding is an additional confirmation of the quantitative analysis of $I_{P L}(\Phi)$.

By substituting into Eq. (31), the calculated dependencies of F vs. $\Phi$ (Fig. 12(b)) we reproduced very well the measured dependencies of PP (Fig. 4(a)). Thus, it is evident that the changes of $F$ are mainly responsible for the variety of YL parameter evolution. Namely, in the case of the structure biased to accumulation, YL is created in the depletion regions of $\mathrm{G} / \mathrm{G}$ interfaces (Fig. 6(a)), in which $F$ decreases vs. $\Phi$, as shown in Fig. 12(b) (curve denoted A). As a result, we observed the redshift of PP with a narrowing of FWHM vs. $\Phi$ (Fig. 3(a)). On the contrary, in the case of the structure biased to depletion, YL is generated in the part of the depletion regions common for $\mathrm{G} / \mathrm{G}$ and $\mathrm{I} / \mathrm{S}$ interfaces (Fig. 6(b)), in which $F$ rises vs. $\Phi$ (Fig. 12(b), curve B). As a consequence, we registered the blueshift of PP with a widening of FWHM vs. $\Phi$ (Fig. 3(b)). However, in the case of the structure biased to deep depletion, YL is created in the depletion region related to the I/S interface (Fig. 6(c)), in which $F$ decreases vs. $\Phi$ (Fig. 12(b), curve C). As a result, we noted the redshift of PP with a narrowing of FWHM vs. $\Phi$ (Fig. 3(c)). Furthermore, within the framework of Eq. (29), we can explain why the variations of YL band shape occur mainly at the band high-energy side, but not at its low-energy side (Fig. 3). Namely, it is evident from Eq. (29) that the highenergy part should be attributed to DAPs having dipole moments approximately parallel to $F(\cos \alpha \approx 1)$ and, on the contrary, the low-energy part of YL band should correspond to DAPs with dipole moments approximately perpendicular to $F(\cos \alpha \approx 0)$. Since the emission energy of DAPs with $\cos \alpha \approx 0$ is almost independent on $F$, therefore we did not observe any visible changes at the lower energy side of the YL band. On the other hand, because the emission energy of DAPs with $\cos \alpha \approx 1$ is dependent on $F$, we observed the marked variations at the band high-energy side.

The above results indicate that YL may come from DAP-type transitions, in which the final state comprises both the Coulomb interaction and strong interaction of the dipole moment with the electric field (see Eq. (29)). Furthermore, the broadening of YL band can be due to the angular distribution of dipole moments with respect to the electric field. It should be noted that the dipole DAP-related recombination mechanism of YL can play a significant role at RT when the flat-band conditions cannot be easily achieved under continuous illumination with typical $\mathrm{HeCd}$ laser fluxes, and thus the band bending of about $0.3-0.6 \mathrm{eV}$ may exist at $\mathrm{GaN}$ surfaces/interfaces. However, this recombination mechanism can be negligible at low temperatures, because under a typical UV illumination of $\mathrm{GaN}$, surface barriers mostly 
disappear due to the strong surface photovoltage effect. ${ }^{44}$ Instead, at low temperatures, YL should be well described in terms of electron-phonon coupling, as it was recently shown by Reshchikov et al., ${ }^{11}$ who observed a zero-phonon line and a fine structure of the YL band.

It is important to stress that the developed phenomenological model, which takes into account the influence of local electric fields on DAP recombination can explain, in a coherent manner, various behaviors of YL. These are, for example, (i) sample dependent variations of PP and FWHM of $\mathrm{YL}^{8}{ }^{8}$ (ii) different dependencies of PP and FWHM vs. $\Phi$, including small and negligible blueshifts of $\mathrm{PP}^{7,12}$ or large blueshifts of $\mathrm{PP},{ }^{45,46}$ (iii) often observed unstable behavior of YL with time under UV illumination, ${ }^{47-49}$ (iv) changes of YL shape after different surface treatment ${ }^{50}$ and $(v)$ rising of YL intensity vs. increase of surface states density, which was observed by Shalish et $a l^{22,23}$ and assigned to the radiative surface states responsible for YL and (vi) cathodoluminescence images registered by Ponce et al. ${ }^{26,51}$ and others ${ }^{52}$ showing that YL is mainly emitted from the near grain boundary regions. It is evident that all the above effects can be attributed, in accordance with our model, to the variations of surface band bending, which are responsible for the changes in dipole energy and carrier distribution in the depletion regions. However, it should also be noted that we do not claim that the previous explanations of the above problems $(i-v i)$ are incorrect but we would just like to show that our model offers an alternative interpretation of these issues.

\section{CONCLUSIONS}

We investigated the $\Phi$-dependent PL at different $V_{G}$ from the GaN-based MIS structure at RT. We found that for different $V_{G}$, various $\Phi$-dependencies of the YL band energy position, FWHM and intensity can be obtained. In particular, we revealed that depending on the applied $V_{G}$, the YL band exhibited a blueshift or a redshift with increasing $\Phi$. In order to explain these results, we developed the phenomenological model, which is based on the solution of the Poisson's equation, current continuity equations, and rate equations with the boundary conditions determined by the interface field strength and nonradiative recombination at semiconductor surfaces and grain boundaries. Our model reproduced well the experimental $\Phi$-dependencies of YL band intensity. It also, predicts that YL arises from the DAP recombination in very limited areas (width of several nanometers) inside depletion regions related to grain/grain interfaces and external crystal surfaces. On this basis, we showed that $V_{G^{-}}$controlled $\Phi$-dependencies of YL peak position and FWHM, can be well explained if we assume that YL is due to DAPtype transitions, in which the final state consists of the Coulomb interaction and strong interaction between the dipole moment of ionized DAP and the depletion region electric field. This recombination mechanism can play a significant role at RT, but should be negligible at low temperatures, where one can expect the reduction of interface barriers under UV illumination due to the strong surface photovoltage effect. Finally, we demonstrated that the proposed phenomenological model, which takes into account the influence of local electric fields on DAP recombination can serve as a coherent explanation of various behaviors of defect related PL.

\section{ACKNOWLEDGMENTS}

One of the authors, M.M., thanks the Research Center for Integrated Quantum Electronics (RCIQE), Hokkaido University, Sapporo, Japan for the postdoctoral fellowship and the members of RCIQE for their kind assistance. M.M. expresses gratitude to Professor Tamotsu Hashizume (RCIQE) for his kind support and discussions, Professor Yunichi Motohisa (RCIQE) for his help in PL experiment and Dr. Zenji Yatabe (Kumamoto University) for TEM images. The work was partially supported by the InTechFun project (UDA-POIG.01.03.01.00-159/08-04) of EU Structural Funds in Poland and the SAFEMOST project (14/ 990/PNN16/0072) within the Visegrad Group (V4)-Japan Joint Research Program on Advanced Materials.

${ }^{1}$ S. Nakamura and G. Fasol, The Blue Laser-Diode GaN Based Light Emitters and Lasers (Springer-Verlag, Berlin, 1997).

${ }^{2}$ H. Morkoc, Handbook on Nitride Semiconductors and Devices, GaNBased Optical and Electronic Devices, Vol. 3 (Wiley, Weinheim, 2009).

${ }^{3}$ J. L. Lyons, A. Janotti, and C. G. Van de Walle, Appl. Phys. Lett. 97, 152108 (2010).

${ }^{4}$ S. G. Christenson, W. Xie, Y. Y. Sun, and S. B. Zhang, J. Appl. Phys. 118, 135708 (2015).

${ }^{5}$ D. Demchenko, I. Diallo, and M. A. Reshchikov, Phys. Rev. Lett. 110, 087404 (2013).

${ }^{6}$ S. Ito, T. Nakagita, N. Sawaki, H. Ahn, M. Irie, T. Hikosaka, Y. Honda, M. Yamaguchi, and H. Amano, Jpn. J. Appl. Phys. 53, 11RC02 (2014).

${ }^{7}$ M. A. Reshchikov, D. O. Demchenko, A. Usikov, H. Helava, and Y. Makarov, Phys. Rev. B 90, 235203 (2014).

${ }^{8}$ M. A. Reshchikov and H. Morkoc, J. Appl. Phys. 97, 061301 (2005).

${ }^{9}$ M. A. Reshchikov, A. Usikov, H. Helava, and Y. Makarov, Appl. Phys. Lett. 104, 032103 (2014).

${ }^{10}$ M. A. Reshchikov, J. Q. Xie, B. Hertog, and A. Osinsky, J. Appl. Phys. 103, 103514 (2008).

${ }^{11}$ M. A. Reshchikov, J. D. McNamara, F. Zhang, M. Monavarian, A. Usikov, H. Helava, Y. Makarov, and H. Morkoc, Phys. Rev. B 94, 035201 (2016).

${ }^{12}$ T. Ogino and M. Aoki, Jpn. J. Appl. Phys., Part 1 19, 2395 (1980).

${ }^{13}$ J. Neugebauer and C. G. Van de Walle, Appl. Phys. Lett. 69, 503 (1996).

${ }^{14}$ J. S. Colton, P. Y. Yu, K. L. Teo, E. R. Weber, P. Perlin, I. Grzegory, and K. Uchida, Appl. Phys. Lett. 75, 3273 (1999).

${ }^{15}$ A. Sedhain, J. Li, J. Y. Lin, and H. X. Jiang, Appl. Phys. Lett. 96, 151902 (2010).

${ }^{16}$ T. Suski, P. Perlin, H. Teisseyre, M. Leszczynsky, I. Grzegory, J. Jun, M. Bockowski, S. Porowski, and T. D. Moustakas, Appl. Phys. Lett. 67, 2188 (1995).

${ }^{17}$ H. M. Chen, Y. F. Chen, M. C. Lee, and M. S. Feng, Phys. Rev. B 56, 6942 (1997).

${ }^{18}$ C. Mizue, Y. Hori, M. Miczek, and T. Hashizume, Jpn. J. Appl. Phys., Part 1 50, 021001 (2011).

${ }^{19}$ M. Matys, B. Adamowicz, and T. Hashizume, Appl. Phys. Lett. 101, 231608 (2012).

${ }^{20}$ B. I. Shklovskii and A. L. Efros, Electronic Properties of Doped Semiconductors (Springer, Berlin, 1984).

${ }^{21}$ A. P. Levanyuk and V. V. Osipov, Usp. Fiz. Nauk. 133, 427 (1981).

${ }^{22}$ I. Shalish, L. Kronik, G. Segal, Y. Rosenwaks, Y. Shapira, U. Tisch, and J. Salzman, Phys. Rev. B 59, 9748 (1999).

${ }^{23}$ I. Shalish, L. Kronik, G. Segal, Y. Shapira, S. Zamir, B. Meyler, and J. Salzman, Phys. Rev. B 61, 15573 (2000).

${ }^{24}$ M. A. Reshchikov, P. Visconti, and H. Morkoc, Appl. Phys. Lett. 78, 177 (2001).

${ }^{25}$ J. I. Izpura, Adv. Condens. Matter Phys. 2013, 597265 (2013). 
${ }^{26}$ F. A. Ponce and D. P. Bour, Nat. London 386, 351 (1997).

${ }^{27}$ W. Shockley and W. T. Read, Phys. Rev. 87, 835 (1952).

${ }^{28}$ K. Colbow, Phys. Rev. 139, A274 (1965).

${ }^{29}$ D. G. Thomas, J. J. Hopfield, and W. M. Augustyniak, Phys. Rev. 140, A202 (1965).

${ }^{30}$ M. Matys, R. Stoklas, J. Kuzmik, B. Adamowicz, Z. Yatabe, and T. Hashizume, J. Appl. Phys. 119, 205304 (2016).

${ }^{31}$ N. Sarkar, S. Dhar, and S. Ghosh, J. Phys.: Condens. Matter 15, 7325 (2003).

${ }^{32}$ F. Binet, J. Y. Duboz, E. Rosencher, F. Scholz, and V. Harle, Appl. Phys. Lett. 69, 1202 (1996)

${ }^{33}$ A. Alkauskas, Q. Yan, and C. G. Van de Walle, Phys. Rev. B 90, 075202 (2014).

${ }^{34}$ J. L. Lyons, A. Alkauskas, A. Janotti, and C. G. Van de Walle, Phys. Status Solidi B 252, 900 (2015).

${ }^{35}$ P. Kamyczek, E. Placzek-Popko, V. Kolkovsky, S. Grzanka, and R. Czernecki, J. Appl. Phys. 111, 113105 (2012).

${ }^{36}$ D. Bisi, M. Meneghini, C. de Santi, A. Chini, M. Dammann, P. Brckner, M. Mikulla, G. Meneghesso, and E. Zanoni, IEEE Trans. Electron Devices 60, 3166 (2013).

${ }^{37}$ A. Domanowska, M. Miczek, R. Ucka, M. Matys, B. Adamowicz, J. Zywicki, A. Taube, K. Korwin-Mikke, S. Gieratowska, and M. Sochacki, Appl. Surf. Sci. 258, 8354 (2012)

${ }^{38}$ M. A. Reshchikov and R. Y. Korotkov, Phys. Rev. B 64, 115205 (2001).

${ }^{39}$ M. A. Reshchikov, Appl. Phys. Lett. 88, 202104 (2006).
${ }^{40}$ M. A. Reshchikov, A. J. Olsen, M. F. Bishop, and T. McMullen, Phys. Rev. B 88, 075204 (2013).

${ }^{41}$ Z. Z. Bandic, P. M. Bridger, E. C. Piquette, and T. C. McGill, Appl. Phys. Lett. 72, 3166 (1998).

${ }^{42}$ K. Kumakura, T. Makimoto, N. Kobayashi, T. Hashizume, T. Fukui, and H. Hasegawa, Appl. Phys. Lett. 86, 052105 (2005).

${ }^{43}$ M. Kato, H. Watanabe, M. Ichimura, and E. Arai, Mater. Res. Soc. Symp. Proc. 831, 107 (2006).

${ }^{44}$ L. Kronik and Y. Shapira, Surf. Sci. Rep. 37, 1 (1999).

${ }^{45}$ M. A. Reshchikov, H. Morkoc, S. S. Park, and K. Y. Lee, Appl. Phys. Lett. 78, 2882 (2001).

${ }^{46}$ M. A. Reshchikov, H. Morkoc, S. S. Park, and K. Y. Lee, Appl. Phys. Lett. 78, 3041 (2001).

${ }^{47}$ M. A. Reshchikov, Y. T. Moon, and H. Morkoc, Phys. Status Solidi C 2, 2716 (2005)

${ }^{48}$ S. A. Brown, R. J. Reeves, C. S. Haase, R. Cheung, C. Kirchner, and M. Kamp, Appl. Phys. Lett. 75, 3285 (1999).

${ }^{49}$ B. J. Ryan, M. O. Henry, E. McGlynn, and J. Fryar, Physica B 340-342, 452 (2003).

${ }^{50}$ R. Cheung, R. J. Reeves, S. A. Brown, E. van der Drift, and M. Kamp, J. Appl. Phys. 88, 7110 (2000).

${ }^{51}$ F. A. Ponce, D. P. Bour, W. Gotz, and P. J. Wright, Appl. Phys. Lett. 68, 57 (1996).

${ }^{52}$ L. J. Brillson, T. M. Levin, G. H. Jessen, A. P. Young, C. Tu, Y. Naoi, F. A. Ponce, Y. Yang, G. J. Lapeyre, J. D. MacKenzie, and C. R. Abernathy, Physica B 273-274, 70 (1999). 